\title{
SENECA: An Attention Support Tool for Context-related Content Learning
}

\author{
Alessia Auriemma Citarella, Luigi Di Biasi, Stefano Piotto, Michele Risi, Genoveffa Tortora \\ Department of Computer Science \\ University of Salerno \\ 84084 Fisciano (SA), Italy \\ \{aauriemmacitarella, ldibiasi, piotto, mrisi, tortora\}@unisa.it
}

\begin{abstract}
In this work, we proposed a tool named SENECA that aims to help the students who follow remote lessons to maintain/capture attention, allowing them to focus on learning led by the context. Among the disadvantages of distance education, especially for subjects who lack awareness, the greatest distractions at home are counted. These distractions cause a movement of the student's attention from the current lesson to disturbing events. For this reason, there is a need to experiment with new solutions also linked to Information Technology (IT) to improve the focused learning during distance education. Our tool's technical idea is to create a real-time summary of the topic treated by the teacher. The system captures the text every five minutes, generates outlines, and scratches them and browses them to eliminate repetitive portions after each survey. On the general generated summary, Natural Language Processing techniques are applied to extract categories and keywords. The latter will show the highlights of the speech.
\end{abstract}

Index terms - Natural Language Processing, Semantic Analysis, Distance Education, Learning, Attention, Content

\section{Introduction}

Into the current pandemic situation generated by the SARS-CoV-2 coronavirus, the educational environment worldwide has to face numerous challenges to continue teaching in schools and universities.

One of the most important aspects of cognitive function is the "ability to keep" relevant information in mind. Working memory is a system dedicated to the maintenance and temporary processing of information during cognitive processes. One of the components is represented by the central executive which carries out the coordination of subordinate systems, coordination of execution of tasks and recovery of strategies and attentional functions of both selection and inhibition [3]. The central executive controls the phono- logical loop which contains verbal and auditory information, the visuo-spatial sketchpad engaged in spatial representation and the episodic buffer which has a limited ability to link information from different sources with spatial and temporal parameters.

Specifically, each attentive act is divided into three phases: the orientation and perception towards the different stimuli; the processing phase that presents the function of selectivity and sustained attention overtime on a task or activity, the shift to move the focus quickly and the ability to pay attention to use the right cognitive resources in different situations; the specific response concerning the input stimuli [4].

Different studies have focused on the impact of technologies on cognitive functions in the present digitized era, both from the perspective of the benefits and disadvantages [38]. Lodge and Harrison [25] stressed as attention is subject to complex dynamics that impact learning, especially in educational contexts. The most important part of a sentence, oral or written, is the focus. Recent articles have demonstrated the importance of marking elements as a guide for better information exchange [24] between speakers and listeners. In particular, these studies argue that focus marking captures the listener's attention to what the speaker considers the most relevant part of the message. At the same time, this method helps the attention to be kept on the marked element allowing its representation [32].

Recent work has addressed the issue of distance education by administering questionnaires to both teachers and pupils. The most variable answers to the questions were also obtained on the degree of students' participation in distance lessons, emphasizing a wide range of behaviors. Furthermore, perception of difficulty during remote lessons was found to be linked to many factors: access to technology, motivation and support with a greater presence of negative experiences [26]. The new educational needs of online teaching and students' changing learning styles limit knowledge transfer comprehensively and effectively. During the detachment of presence foreseen by distance teach- 




Figure 1: A standard bidirectional media streaming.

ing, many dysfunctional behaviors can be generated, such as the loss of interest, attention and motivation for psychophysical causes and non-adaptation to an abnormal situation. Compared to the standard educational environment, distance learning has a disadvantage in terms of distractions. It is an isolated experience in which there is no direct communication that makes participation much more active [14]. It would be useful to overcome the difficulties of maintaining a student's attention, regardless of the need or not of a situation that requires the use of tools for distance learning. Overcoming these issues would help refine each pupil's strategic learning styles and ensure a metacognitive self-assessment approach on one's limits and abilities, supported by technology. Recent meta-cognitive skills also include a student's ability to become aware of his or her ability to "learning how to learn". This ability means recognizing and then consciously applying appropriate behaviors and strategies useful for a more effective learning process [29].

This paper proposes a Distributed Multimedia System for support learning, designed to face the loss of attention during distance education. The purpose of the system is to be able to reawaken or maintain attention to the context (topic) that is being experienced during the activity in progress (in real time) to reduce the negative effect of distractions. The system also aims to provide the possibility of an in-depth analysis at the end of the lesson through autogenerated hyperlinks to lesson-related content. The architecture proposed rely on Speech-to-text, Natural Language Processing (NLP), Text Summarization [18] and Semantic Analysis technology.

This document is organized as follows. In Section 2, we described the most important related works about the technological systems that help in learning. In Section 3 we introduced the used methodologies. In Section 4 we have highlighted the working hypotheses on which we based our work. In Section 5 we presented the system architecture and in Section 6 we have detailed the performed experiments and the related results (Section 7). Finally, in Section 8 we will discuss research directions and future development of our work.

\section{Related works}

In this section, we present some related works about the use of semantic and NLP analysis technologies. Specifically, we will discuss different application contexts of these techniques. Most of a student's effort is to transfer information from working memory to long-term memory to acquire and memorize key concepts. Two strategies can be used: dual coding and chunking [10]. In cognitive psychology, a chunck is nothing more than a unit of information, and chunking is the operating mode in which this unit of data is recovered. When faced with new knowledge, the individual can grasp the relative chunk of information and bring it back to light later when recalling a similar situation or concept. Then, the initial piece can be expanded into more complex pieces following the management control and understanding of the flows of one's knowledge [35].

The standard structure of a Distance Educational System can be generalized as reported in Fig. 1. This kind of system relies on the classic bidirectional multimedia connection, like the common video-chat system based on SIP/VOIP system, such as Microsoft Teams [23]. However, a Distance Educational System supports multiple bidirectional connections between students and teachers (or teachers) and allows channel moderation.

Nowadays, the cloud's audio/video stream transfer services are implemented by the major world providers (Amazon, Microsoft, Zoom). A teacher can teach remotely by transmitting an audio/video stream from their home to one of these providers. Then these last provide a broadcast service to the students.

NLP techniques have been widely used in intelligent tutoring systems that helped acquire content knowledge [8]. For example, in Guzmán-García et al. [21], the analysis of the surgeons' speaking taken into the operating room through NLP techniques is proposed to obtain a deeper vision of intraoperative decision-making processes. This study aimed to develop a method of recognizing and evalu- 


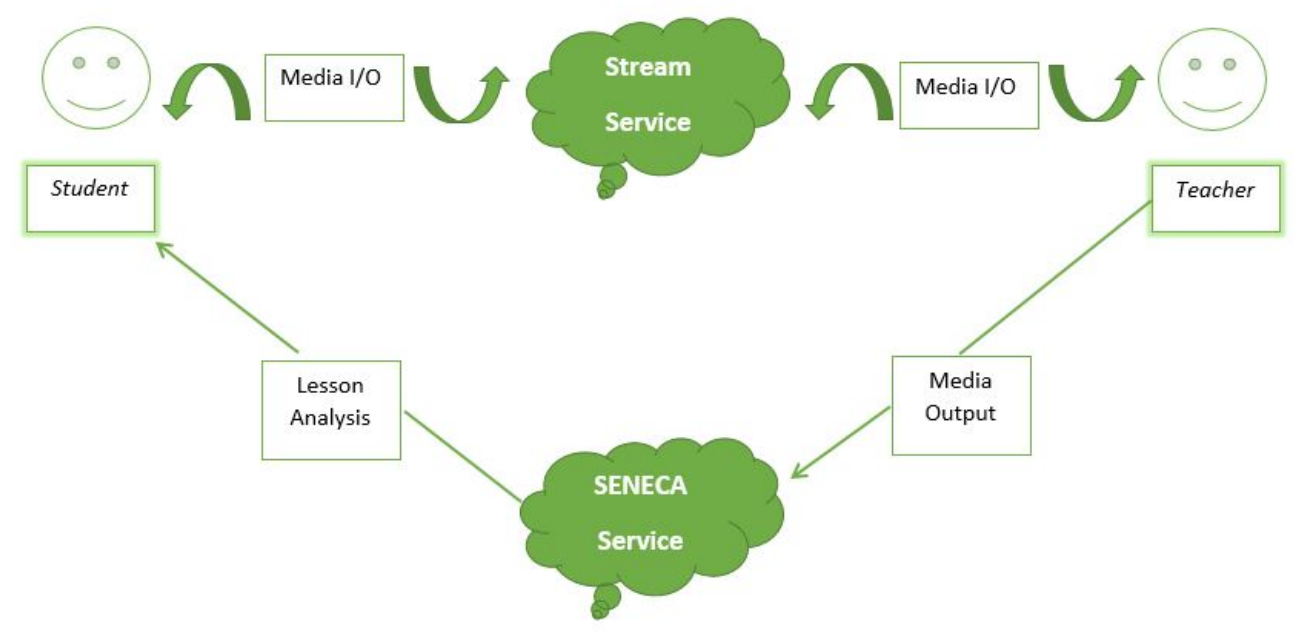

Figure 2: The additive layer for multimedia analysis.

ating the various surgical phases and developing a workflow comparable with the framework of the procedure to improve surgical learning in The Educational Operating Room.

Recent studies have highlighted how to identify the main contents to better understand the topic, especially in students with cognitive deficiency, attention, or memory. The ability to take advantage of text summarization techniques by explaining the main idea allows students to interface with the limits of their working memory and have a tool to overcome their difficulties [34].

Today, many educational and academic institutions benefit from the Learning Management Systems (LMS) to support and improve teaching processes [15][19]. Most LMS is software application systems that allow teachers to manage and deliver educational courses [2]. One of the requirements for the success of distance education is traceable in the self-management of learning which is the starting point for self-discipline in autonomous learning [36].

In 2019, Cobos et al. [12] have developed EdX-CAS, a content analyzer system for edX MOOCs, using NLP techniques for the Spanish language. The tool takes in input the video transcripts of the courses, with which users can interact specifically. It allows students to extract the text's main terms, the vector representation for each of the terms in the text, the linguistic diversity to understand how many different words are used, indications on the subjective opinion on the text and the representation with word clouds. The edXCAS tool is oriented to Sentiment Analysis Opinion Mining for Detecting Subjectivity and Polarity Detection in Online Courses related to Madrid's Universidad Autónoma.

On the topic of the educational distance imposed by the Covid-19, to support students in self-training, a chatbot was proposed using NLP techniques [16]. The proposed solu- tion involves sending a message to Moodle [1] by the student. An associated plugin tries to understand the text received and returns feedback. Based on the degree of assessment achieved by the student, the chatbot provides recommendations on the chapters for which the evaluation is not sufficient. The system presupposes the memorization of the evaluation outcomes of the student who are accessible to teachers. The chatbox, in this context, acts as a tutor and allows us to fill the gaps of the students.

\section{Methods}

We propose a new tool called SENECA (Support lEarning coNtEnt Context Attention), which involves using a new layer dedicated exclusively to analyzing the audio/video streams generated by the "teachers". Our purpose is to maintain the student focus or a rapid return to it by implementing this new layer. The proposed architecture is to be considered feasible for real-time distance lessons and not to the MOOC [5] or the on-demand recorded lessons. We did not consider the capabilities related to file upload, file sharing and homework as an added values.

The new layer is identified in the Fig. 2 as Seneca Service. In this proposal, we refer only to real-time audio/video streams, i.e., not recorded lessons held at a distance. The real-time component's presence allows us to immerse ourselves in a learning context susceptible to disturbances that distract the individual student.

SENECA's main goal is to help students avoid loss of concentration by providing multiple information that can allow students to maintain focus on the argument or bring attention back to the context in the event of distractions. Another important goal is to favor applying many text analysis 
techniques to improve the learning quality of the topic under study and integrate it, providing additional information that can be used at the end of the lesson to recover information further.

\section{Working hypothesis}

In this section, we aim to highlight some working hypotheses that allow us to have a more understandable general view of the SENECA tool's starting idea:

a) The real-time audio/video stream (from here called STREAM) generated by a remote lesson can be split into two unique sub-streams: VIDEO flow, containing the video frames and AUD IO flow containing audio buffer.

b) The VIDEO flow will contain information from slides or, in any case, projected material to provide a conceptual map to students.

c) The AUDIO flow will contain the lesson audio, and it is expected to add information on both the context under study and in-depth study (as well as student questions or others).

In this context, we assume that the VIDEO stream contains information already summarized on the subject. In our experiment, we considered the data extracted from VIDEO as already cleaned. On the other hand, the presence of heterogeneous data in AUDIO streams will require a more accurate analysis of the content.

The extracted data from VIDEO and AUDIO is referred following as WORD STREAMS.

\section{System architecture}

We designed a prototype architecture based on a pipeline approach, like Microsoft DirectShow ${ }^{1}$ or ffmpeg ${ }^{2}$. In SENECA each computational block is called Filter.

An overview of a complete SENECA architecture is shown in Fig.3. For this proposal, we implemented only the following filters: SPLIT, OCR, STT, SUMMARY and SEMANTICS.

The filters are defined as following:

$$
\begin{gathered}
S P L I T(S T R E A M) \rightarrow\{A U D I O, V I D E O\} \\
O C R(V I D E O) \rightarrow\{W S\} \\
S T T(A U D I O) \rightarrow\{W S\}
\end{gathered}
$$

\footnotetext{
${ }^{1}$ https://docs.microsoft.com/en-us/windows/win32/directshow/directshow

${ }^{2}$ https://ffmpeg.org/developer.html
}

$$
\begin{aligned}
& S U M M A R Y(W S, G L S) \rightarrow\{N E W G L S\} \\
& \text { SEMANTIC }(G L S) \rightarrow \text { SUGGESTIONS }
\end{aligned}
$$

The SPLIT filter takes as input the STREAM and splits it into two separate flows, called AUDIO and VIDEO.

The OCR technique allows the detection and extraction of text from images [28]. The SENECA OCR Filter takes as input a single frame video at a time. We used the videocr python module (v. 0.1.6) ${ }^{3}$ for our experiment purpose. That module lies on Tesseract OCR 4.1.1. ${ }^{4}$. This filter analyzed each video frame from the pipeline and stored the detected text (handwritten and block letters) into a word stream (WS). Each word stream was enqueued into the next pipeline filter.

In SENECA, the STT filter performs a speech-to-text routine. Speech-to-text is a technique that allows the detection and the extraction of phrases from an audio flow WS [11]. Probably, the most commonly known example is Amazon Alexa or Google Assistant. Into our prototype, we used the Google Cloud Speech API https://cloud.google.com/speech-to-text/. For each audio frame extracted by the SPLIT, the STT filter generated a word-stream (WS) that was enqueued to the NPL filter.

One of the project goals is to provide a way to regain the attention on the topic focus after a distraction. In SENECA, one of the tips is to allow users to summarize the lesson in real-time. As shown in figure 3, the media flow (as an example, a streamed lesson) comes into the SPLIT filter that separates audio from video. For each video frame extracted, the OCR extracts the detected phrases, and STT makes the same action on the audio frame. In particular, these word streams came into the next filter into SUMMARY filter that is a delegate to create partial summaries using the word streams that came into the filter. Into our prototype, the SUMMARY filter computes a summary for the WSs using MEAD [18]. These multiple summaries are merged every 5 minutes into the Global Lesson Summary (GLS) that is processed again by MEAD. We have chosen the five minutes interval using the mean lesson length. Consequently, SENECA builds and refreshes a GLS by using SUMMARY filter output for each real-time lesson. Into our prototype, GLS is composed of phrases generated by applying MEDA text summarization algorithm on WSs.

The SEMANTIC filter extracts the MEF from the GLS every time a new GLS is deployed from the SUMMARY filter. We identify with the term MEF or Most Expressed Features of a string $\mathrm{S}$, the dictionary $\mathrm{D}(\mathrm{S})$ of all possible $k$-mers extracted from $\mathrm{S}$, using substrings length between $\mathrm{m}$

\footnotetext{
${ }^{3}$ https://pypi.org/project/videocr/

${ }^{4}$ https://github.com/tesseract-ocr/tesseract
} 


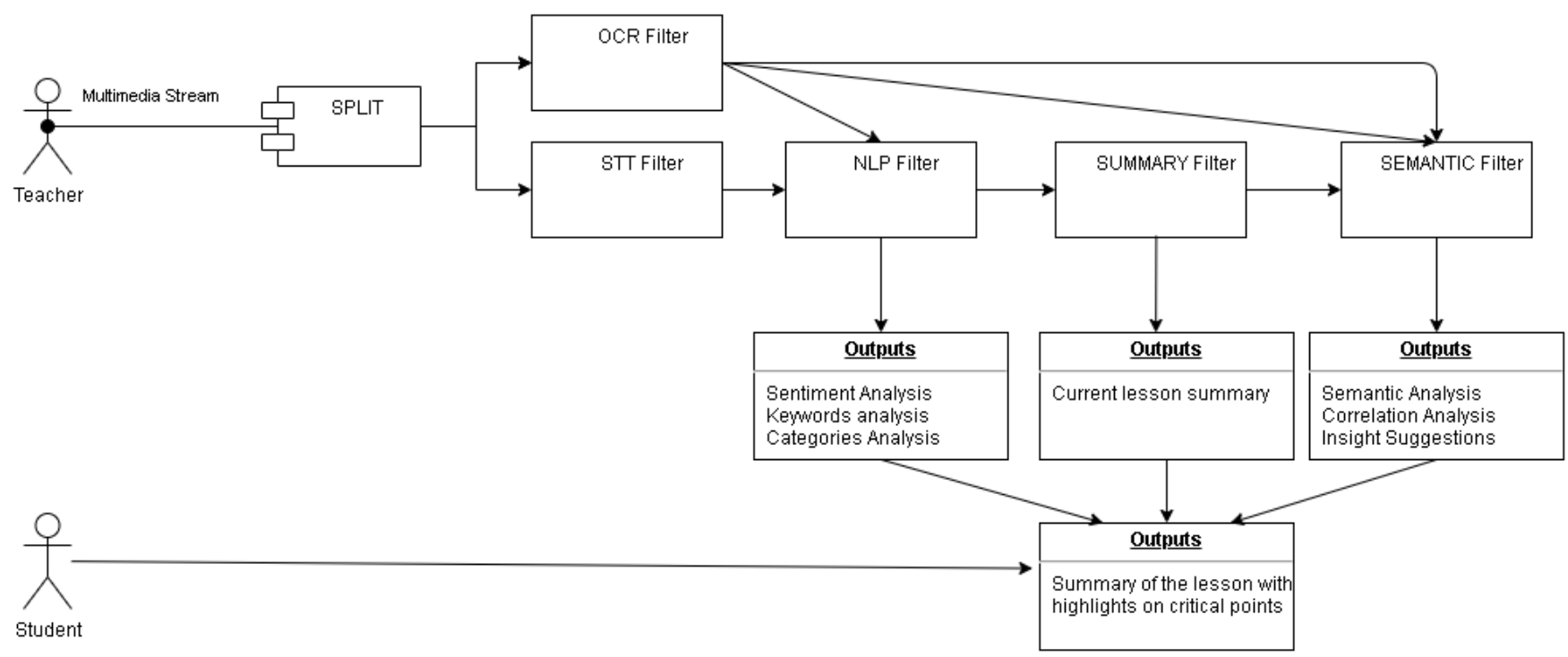

Figure 3: A basic SENECA module.

Table 1: Partial subset of lessons MEF.

\begin{tabular}{cc}
\hline Topics & MEFs \\
\hline Cancer & Smoking, Colon Cancer, Surgery, Risk Factor \\
\hline Diabetes & Beta Cell, Interleukin, Inflammatory, Physic \\
\hline Evolution & Selection, Heritability, Billion Years, Coevolution \\
\hline Terrorism & Terror, Povery, Success, Politican \\
\hline Chemistry & Compound, Energy, Element, Electron \\
\hline
\end{tabular}

and $\mathrm{M}$. The dictionary is ordered in a decreasing way, compared to the number of occurrences of each k-mers. The MEFs represent the object's functional parts, such as words or portions of sentences of a text repeated several times. SEMANTIC is designed to use the MEF for probing one or more scientific databases to make suggestions. In particular, it performs a combined NO-SQL Alignment-free search into the pre-processed PUBMED database (see next paragraph). For each GLS, SEMANTIC can extract the candidate literature papers indexed by MEF. It uses two metrics to compute (see experiment one) the suggested papers based on the semantic distance between GLS and candidate paper set.

For this prototype, we used the PUBMED database [9]. We execute the SEMANTIC filter on the entire PUBMED dataset, and we have extracted the $\mathrm{MEF}$ s, using substrings length interval between $\mathrm{m}=3$ and $\mathrm{M}=15$.

Due to the PUBMED dataset size, we used Amazon EC2 and Amazon RDS services [30] to distribute MEFs extraction and storage.

\section{Experiment Execution}

We simulated real-time lessons by using public videos from Coursera ${ }^{5}$. We selected five free courses, recovering from each it, the text subscription using STT. The main topics of the lessons are Cancer; Diabetes; Evolution; Terrorism, Chemistry.

We implemented two experiments. Our goal was to study the SUMMARY and SEMANTIC filter performances.

\subsection{First Experiment}

Into the first experiment, we sent all the entire lessons (5 merged videos per lesson) into the SENECA pipeline, sequentially, to compute separate GLS outputs for each entire lesson. Also, we sent each video (one at a time, not merged) into the pipeline to generate the GLS for each video.

We wanted to study if the SEMANTIC filter was able to discriminate between lesson topics. We applied the SE-

\footnotetext{
${ }^{5}$ https://www.coursera.org/
} 

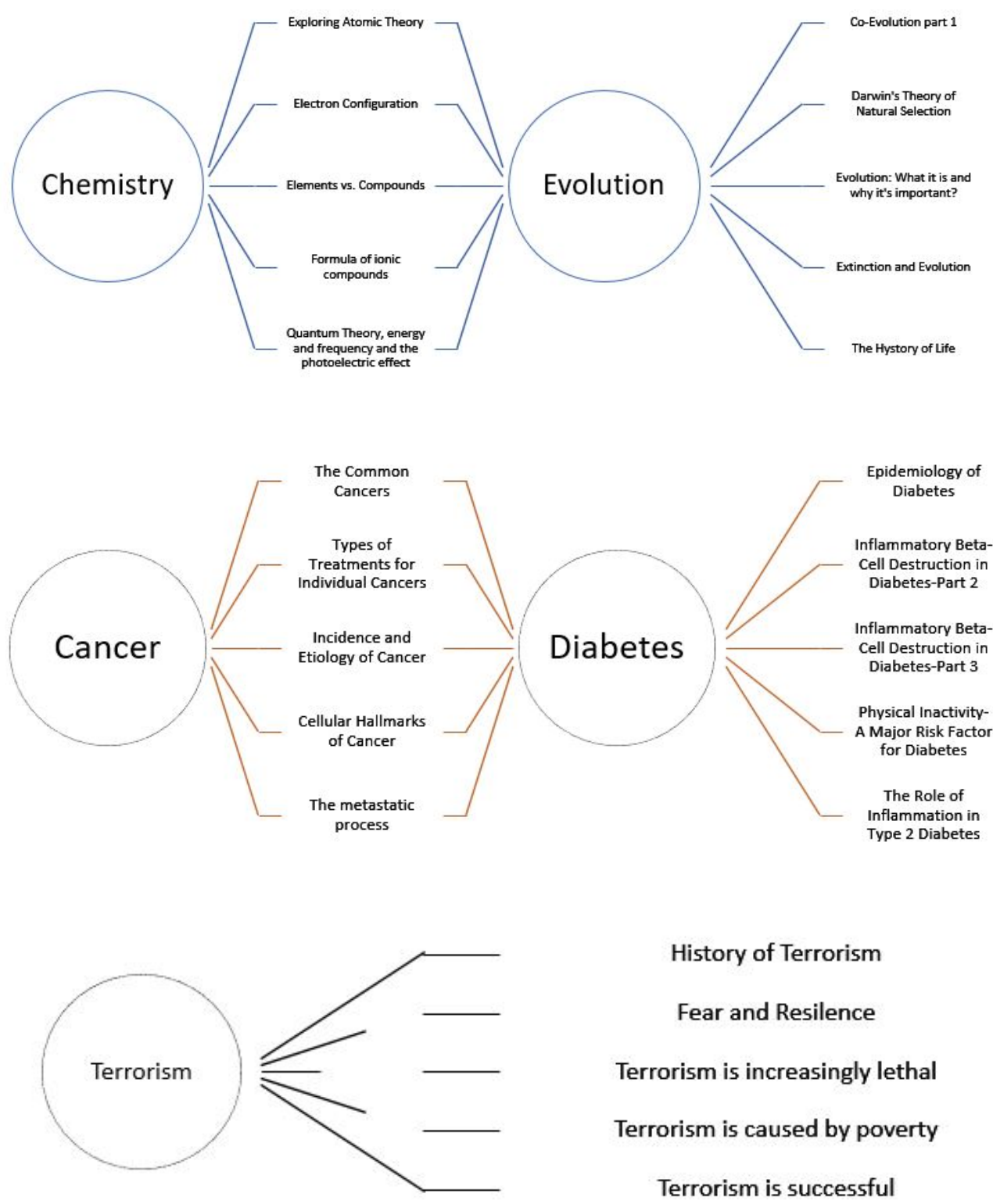

Figure 4: Lesson Topics clustering using Jaccard distance.

MANTIC filter on each GLS to extract the MEFs for each of them.

Using the MEFs, we were able to build two distance matrices. We used two different distance metrics. The distances were calculated using the Jaccard Index [31] (see Eq. 1), and by Szymkiewicz-Simpson coefficient (SSC) [39] (see Eq. 2).

Both metrics allow us to compute the similarity between pair of MEF dictionary. SSC is often identified as the "overlay coefficient."
The Jaccard index is defined as:

$$
J(A, B)=\frac{|A \cap B|}{|A \cup B|}
$$

where $A$ and $B$ are two different datasets, whilst the $|\bullet|$ operator computes the size of a set. In particular, the Jaccard index is represented as the size of the intersection divided by the size of the union of the datasets.

Given the two dictionaries $A$ and $B$, the overlap coefficient is a measure that returns the overlap between them, 

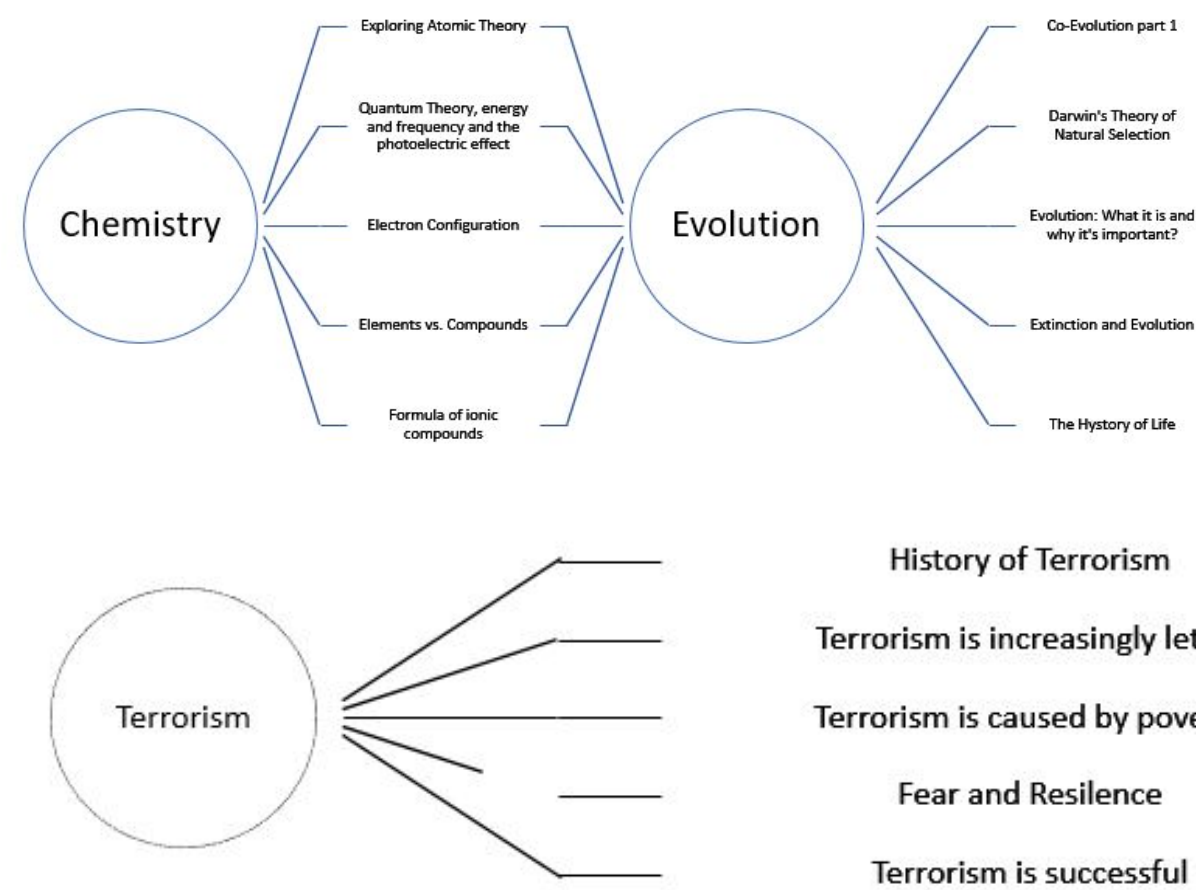

History of Terrorism

Terrorism is increasingly lethal

Terrorism is caused by poverty

Fear and Resilence

Terrorism is successful

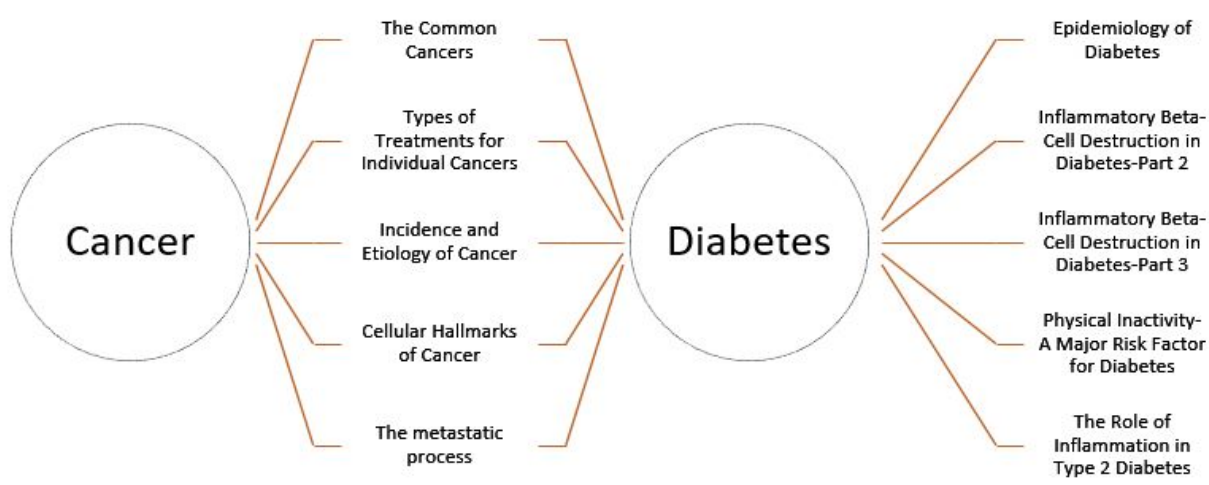

Figure 5: Lesson topics clustering using SSC.

and it is defined as the intersection divided by the smaller of the size of the two sets, as shown in Eq. 2.

$$
S S C(A, B)=\frac{|A \cap B|}{\min (|A|,|B|)}
$$

We used the dictionaries and the distance matrices to extract the most expressed keywords and cluster the GLS.

\subsection{Second Experiment}

Into the second experiment, we sent each lesson, one at a time, to the pipeline to generate its GLS. For each GLS, we applied the SEMANTIC filter to extract the related MEFs to use them as probes for the subsequent insights.

We applied Jaccard and SSC metrics between each GLS and the PUBMED-MEF dataset.

We computed ten distance matrices from which we picked the first ten (see table 2) most similar results for each GLS. We were forced to used Apache Spark [37] to distribute this job across multiple slaves due to the heavy memory requirements of these tasks. 
Table 2: Partial subset of suggested papers and their distance score.

\begin{tabular}{ccc}
\hline Paper ID & Author & Value \\
\hline$P 1$ & Belsky et al. [7] & 0.91 \\
\hline$P 2$ & Huang et al. [22] & 0.81 \\
\hline$P 3$ & Wu [41] & 0.93 \\
\hline$P 4$ & Gaitanidis et al. [17] & 0.83 \\
\hline$P 5$ & Bauer et al. [6] & 0.88 \\
\hline$P 6$ & Wang et al. [40] & 0.95 \\
\hline$P 7$ & Mays et al. [27] & 0.94 \\
\hline$P 8$ & Schuck et al. [33] & 0.93 \\
\hline$P 9$ & Guo et al. [20] & 0.92 \\
\hline$P 10$ & Corsi et al. [13] & 0.90 \\
\hline
\end{tabular}

\section{Experimental Results}

The Table 1 shows the first four MEFs for each lesson. The SEMANTIC filter was able to detect keywords related to lesson context. Due to the GLS and MEF definitions, the MEF dictionaries contain up to $140 \mathrm{~K}$ kmers for each GLS. We reported the most expressed that refer to complete word.

The results of topics clustering are available in Fig. 4 with the Jaccard index and Fig. 5 with the overlap coefficient.

We identified the topics which are part of the same branch of the tree with the same color and the subgroups of each topic using different colors. SEMANTIC separated the five treated topics well, with just little differences in lesson aggregation levels. Specifically, it is interesting to note how the system is able to cluster together the Cancer and Diabetes and Chemistry with Evolution topics.

In the second experiment, we used the extracted MEFs as if they represented 'tags' to recover suggested papers. For convenience, we have used only the MEFs of the lessons on the topics Cancer to show the results.

SEMANTIC identified 274 correlates documents recovered by PUBMED-MEF dataset. For example, in Table 2 we showed the first ten recovered papers with a score value greater than 0.80. In Fig. 6, we represented the position of suggested papers graphically compared to the target query, keeping in mind that the score of the distance from the target query is representative of the similarity between the set of MEFs of the Cancer topic and the individual retrieved papers.

\section{Conclusions}

The change to the basis of remote teaching is the transition from traditional education to smart education. The teacher is responsible for managing class to be studentcentered, which involves greater responsibility and aware- ness of their limits and potential in self-learning behind one screen. It is not always possible. Disturbing factors related to the surrounding environment may disrupt the attention of the student. In an era in which new teaching tools are proposed, managing personal learning is also changed. One of the disadvantages of remote education is the different distractions that can intervene in maintaining the focus. In this work, we have proposed a tool that aims to maintain students' attention on topics covered in the teacher's lessons. The system works in real-time and allows us to generate further knowledge thanks to the possibility of an in-depth study by hyperlinks of treated topics. The preliminary experimentation suggests that the framework can provide insight and help regain attention on the lesson focus. Experimentation in real classes should be performed to understand the framework's impact on student attention in remote lessons. Among the future directions' objectives, we expect to explore techniques that improve text summarization and the extension of architecture for multilingual texts. An additional module for the real-time creation of concept maps may be provided.

\section{References}

[1] A. Al-Ajlan and H. Zedan. Why Moodle. In Procs. of the 12th IEEE International Workshop on Future Trends of Distributed Computing Systems, pages 58-64. IEEE, 2008.

[2] N. A. Alias and A. M. Zainuddin. Innovation for better teaching and learning: Adopting the learning management system. Malaysian Online Journal of Instructional Technology, 2(2):27-40, 2005.

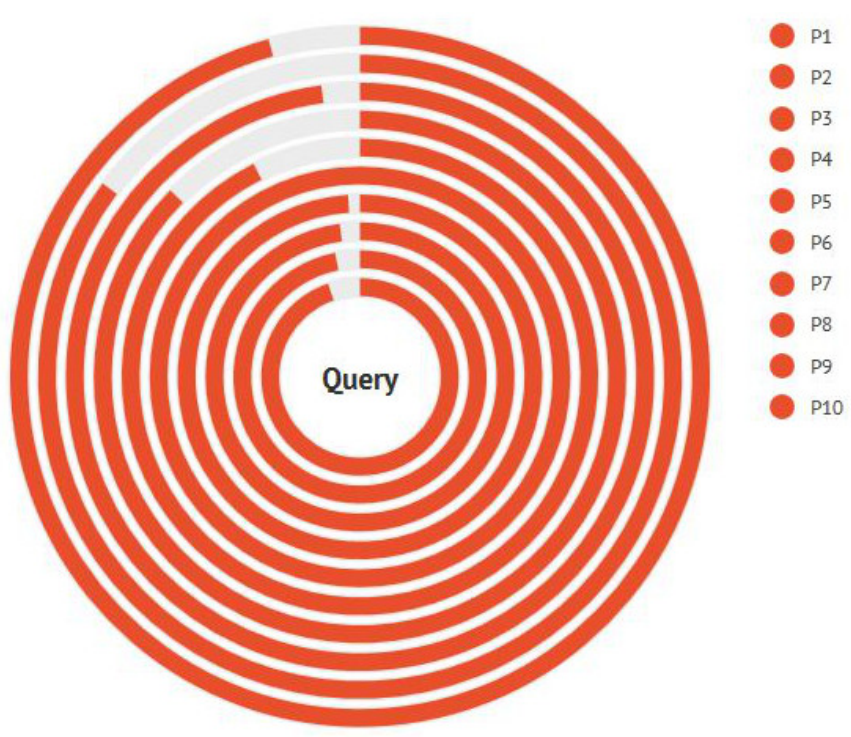

Figure 6: Position of suggested papers compared to the target query. 
[3] A. Baddeley. Exploring the central executive. The Quarterly Journal of Experimental Psychology - Section A, 49(1):528, 1996.

[4] A. Baddeley. The episodic buffer: a new component of working memory? Trends in cognitive sciences, 4(11):417423, 2000.

[5] J. Baggaley. Mooc rampant. Distance Education, 34(3):368-378, 2013.

[6] M. Bauer, E. Morales-Orcajo, L. Klemm, R. Seydewitz, V. Fiebach, T. Siebert, and M. Böl. Biomechanical and microstructural characterisation of the porcine stomach wall: Location-and layer-dependent investigations. Acta Biomaterialia, 102:83-99, 2020.

[7] D. W. Belsky, T. E. Moffitt, T. B. Baker, A. K. Biddle, J. P. Evans, H. Harrington, R. Houts, M. Meier, K. Sugden, B. Williams, et al. Polygenic risk and the developmental progression to heavy, persistent smoking and nicotine dependence: Evidence from a 4-decade longitudinal study. JAMA Psychiatry, 70(5):534-542, 2013.

[8] J. Burstein. Opportunities for natural language processing research in education. In Procs. of the International Conference on Intelligent Text Processing and Computational Linguistics, pages 6-27. Springer, 2009.

[9] K. Canese and S. Weis. Pubmed: The bibliographic database. In The NCBI Handbook [Internet]. 2nd edition. National Center for Biotechnology Information (US), 2013.

[10] S. L. Chew and W. J. Cerbin. The cognitive challenges of effective teaching. The Journal of Economic Education, 52(1):17-40, 2021.

[11] C. C. Chiu, T. N. Sainath, Y. Wu, R. Prabhavalkar, P. Nguyen, Z. Chen, A. Kannan, R. J. Weiss, K. Rao, E. Gonina, N. Jaitly, B. Li, J. Chorowski, and M. Bacchiani. State-of-the-art speech recognition with sequenceto-sequence models. In Procs. of the IEEE International Conference on Acoustics, Speech and Signal Processing (ICASSP), pages 4774-4778, 2018.

[12] R. Cobos, F. Jurado, and A. Blázquez-Herranz. A content analysis system that supports sentiment analysis for subjectivity and polarity detection in online courses. IEEE Revista Iberoamericana de Tecnologías Del Aprendizaje, 14(4):177-187, 2019.

[13] D. J. Corsi, C. K. Chow, S. A. Lear, S. Subramanian, K. K. Teo, and M. H. Boyle. Smoking in context: A multilevel analysis of 49,088 communities in Canada. American Journal of Preventive Medicine, 43(6):601-610, 2012.

[14] J. Cowan. The advantages and disadvantages of distance education. Distance Education for Language Teachers: A UK Perspective, pages 14-20, 1995.

[15] R. Francese, C. Gravino, M. Risi, G. Scanniello, and G. Tortora. Using project-based-learning in a mobile application development course-an experience report. Journal of Visual Languages \& Computing, 31:196-205, 2015.

[16] K. Gaglo, B. M. Degboe, G. M. Kossingou, and S. Ouya. Proposal of conversational chatbots for educational remediation in the context of Covid-19. In Procs. of the 23rd International Conference on Advanced Communication Technology (ICACT), pages 354-358. IEEE, 2021.

[17] A. Gaitanidis, N. Machairas, M. Alevizakos, C. Tsalikidis, A. Tsaroucha, and M. Pitiakoudis. Predictive nomograms for synchronous liver and lung metastasis in colon cancer. Journal of Gastrointestinal Cancer, pages 1-7, 2019.

[18] M. Gambhir and V. Gupta. Recent automatic text summarization techniques: A survey. Artificial Intelligence Review, 47(1):1-66, 2017.

[19] C. Gravino, M. Risi, G. Scanniello, G. Tortora, and R. Francese. Supporting mobile development project-based learning by software project and product measures. In Procs. of the 22nd International Conference on Distributed Multimedia Systems (DMS), pages 41-47, 2016.

[20] Q. Guo, J. B. Unger, P. H. Palmer, C.-P. Chou, and C. A. Johnson. The role of cognitive attributions for smoking in subsequent smoking progression and regression among adolescents in China. Addictive Behaviors, 38(1):1493-1498, 2013.

[21] C. Guzmán-García, M. Gómez-Tome, P. Sánchez-González, I. Oropesa, and E. J. Gómez. Speech-based surgical phase recognition for non-intrusive surgical skills' assessment in educational contexts. Sensors, 21(4):1330, 2021.

[22] X. Huang, Y. Zou, L. Lian, X. Wu, X. He, X. He, X. Wu, Y. Huang, and P. Lan. Changes of $\mathrm{T}$ cells and cytokines TGF- $\beta 1$ and IL-10 in mice during liver metastasis of colon carcinoma: Implications for liver anti-tumor immunity. Journal of Gastrointestinal Surgery, 17(7):1283-1291, 2013.

[23] M. Hubbard and M. J. Bailey. Mastering Microsoft Teams. Mastering Microsoft Teams. https://doi. org/10.1007/978-14842-3670-3, 2018

[24] T. Káldi and A. Babarczy. Linguistic focus guides attention during the encoding and refreshing of working memory content. Journal of Memory and Language, 116:104187, 2021.

[25] J. M. Lodge and W. J. Harrison. Focus: Attention science: The role of attention in learning in the digital age. The Yale Journal of Biology and Medicine, 92(1):21, 2019.

[26] M. W. Marek, C. S. Chew, and W.-c. V. Wu. Teacher experiences in converting classes to distance learning in the Covid-19 pandemic. International Journal of Distance Education Technologies (IJDET), 19(1):40-60, 2021.

[27] D. Mays, G. Luta, L. R. Walker, and K. P. Tercyak. Exposure to peers who smoke moderates the association between sports participation and cigarette smoking behavior among non-white adolescents. Addictive Behaviors, 37(10):11141121, 2012.

[28] J. Memon, M. Sami, R. A. Khan, and M. Uddin. Handwritten optical character recognition (OCR): A comprehensive systematic literature review (SLR). IEEE Access, 8:142642$142668,2020$.

[29] C. M. Mowling and S. K. Sims. The metacognition journey: Strategies for teacher candidate exploration of self and student metacognition. Strategies, 34(2):13-23, 2021.

[30] T. Mufti, P. Mittal, and B. Gupta. A review on Amazon web service (AWS), Microsoft azure \& Google cloud platform (GCP) services. European Union Digital Library, 2021.

[31] S. Niwattanakul, J. Singthongchai, E. Naenudorn, and S. Wanapu. Using of Jaccard coefficient for keywords similarity. In Procs. of the International Multiconference of Engineers and Computer Scientists, pages 380-384, 2013.

[32] A. J. Sanford, A. J. Sanford, J. Molle, and C. Emmott. Shallow processing and attention capture in written and spoken discourse. Discourse Processes, 42(2):109-130, 2006. 
[33] K. Schuck, R. Otten, R. C. Engels, and M. Kleinjan. The role of environmental smoking in smoking-related cognitions and susceptibility to smoking in never-smoking 9-12 year-old children. Addictive Behaviors, 37(12):1400-1405, 2012.

[34] A. Shelton, C. J. Lemons, and J. Wexler. Supporting main idea identification and text summarization in middle school co-taught classes. Intervention in School and Clinic, 56(4):217-223, 2021.

[35] H. A. Simon. How big is a chunk?: By combining data from several experiments, a basic human memory unit can be identified and measured. Science, 183(4124):482-488, 1974.

[36] P. J. Smith, K. L. Murphy, and S. E. Mahoney. Towards identifying factors underlying readiness for online learning: An exploratory study. Distance Education, 24(1):57-67, 2003.

[37] A. Spark. Apache spark. Retrieved January, 17, 2018.

[38] A. Venditti, F. Fasano, M. Risi, and G. Tortora. The importance of interaction mechanisms in blended learning courses involving problem solving e-tivities. In Procs. of the 13th International Conference on Digital Information Management (ICDIM), pages 124-129, 092018.

[39] M. Vijaymeena and K. Kavitha. A survey on similarity measures in text mining. Machine Learning and Applications: An International Journal, 3(2):19-28, 2016.

[40] M.-P. Wang, S.-Y. Ho, W.-S. Lo, and T.-H. Lam. Smoking family, secondhand smoke exposure at home, and nicotine addiction among adolescent smokers. Addictive Behaviors, 37(6):743-746, 2012.

[41] C.-Y. Wu. Initiatives for a healthy stomach. Current Treatment Options in Gastroenterology, 17(4):628-635, 2019. 